\title{
Investigations and control measures following a non- travel-associated case of toxigenic Cornyebacterium diphtheriae, London, United Kingdom, December 2009-January 2010
}

S Perkins (shona.perkins@hpa.org.uk)1, R Cordery ${ }^{1}$, G Nixon ${ }^{1}$, A Abrahams ${ }^{1}$, J Andrews ${ }^{2}$, J White A A Efstratiou $^{3}$, S Anaraki $^{1}$

1. North East and North Central London Health Protection Unit, United Kingdom

2. Whittington Hospital NHS Trust, London, United Kingdom

3. Centre for Infections, Health Protection Agency, London, United Kingdom

This article reports the investigation and control measures undertaken following the identification of a toxigenic strain of Cornyebacterium diphtheriae var gravis, designated ribotype 'Minsk', in a partially vaccinated teenager born in the United Kingdom with no recent history of travel or known contact with a case of diphtheria or a carrier. This case highlights the need for ongoing work to improve vaccine uptake rates to ensure children receive all scheduled vaccinations.

\section{Introduction}

Diphtheria is an acute bacterial disease that primarily affects the respiratory mucosa and skin, but more rarely has also been found in other mucous membranes such as conjunctivae, vagina and ear [1]. Three Corynebacterium spp. can potentially produce diphtheria toxin: $C$. diphtheriae, which has four biotypes: gravis, mitis, intermedius and belfanti, C. ulcerans and C. pseudotuberculosis. Toxin production occurs when bacteria are infected by corynebacteriophage containing the toxin gene tox [1].

In the United Kingdom (UK) toxigenic $C$. diphtheriae has become very rare (Table) due to national immunisation that has been in place since 1942 [2]. The small number of isolates identified are most commonly associated with travel to endemic countries. The $C$. diphtheriae biotype gravis "Minsk" ribotype, although uncommon in the UK, has been previously isolated in a UK immigrant during the $1990 \mathrm{~s}$. This ribotype was originally observed among diphtheria cases in Belarus during the period from 1993 to 2000 . It was only prevalent in Belarus during the height of the diphtheria epidemics in the Newly Independent States of the former Soviet Union during the 1990s [Personal communication: Streptococcus and Diphtheria Reference Unit, Centre for Infection, Health Protection Agency, UK].

The last death in the UK from diphtheria was in an unvaccinated school-aged child in 2008 who had no recent history of travel. A public health investigation did not identify a source of infection [4]. A toxigenic C. diphtheriae var mitis was grown from a diagnostic bronchoalveolar lavage sample.

The UK vaccination schedule includes a primary course of diphtheria-containing vaccine given at two, three, and four months of age, with a pre-school booster given between three years and four months and five years of age. A school leavers' booster is then scheduled between the ages of 13 and 18 years [5].

\section{Case report}

On 31 December 2009 the case presented to a London hospital accident and emergency unit with a five-day history of severe sore throat, pustular tonsils and abdominal pain in the right upper quadrant. There was no history of travel and a pseudomembrane was not seen on clinical examination. A throat swab was taken. The case was admitted to a bay on a paediatric ward and treated with intravenous benzylpenicillin.

On 2 January 2010 a Monospot test was positive for Epstein-Barr virus (EBV). At this point intravenous antibiotics were stopped and a differential diagnosis of glandular fever was made. The case remained in hospital for four days (nine days after onset of disease) and was discharged on 4 January 2010 following clinical recovery. On 5 January 2010 the throat swab culture had grown group C beta-haemolytic Streptococcus.

The North East and North Central Health Protection Unit (HPU) were notified by the hospital medical microbiology registrar on 7 January 2010 that the throat swab had also grown $C$. diphtheriae. The sample was urgently couriered to the Health Protection Agency's (HPA) Streptococcus/Diphtheria Reference Unit to assess toxigenicity. It was identified as a toxigenic 
C. diphtheriae var gravis strain by conventional and molecular methods [6].

On the evening of 8 January 2010 the HPU informed the family doctor of the results and advised of the need for medical assessment of the case and any close household contacts. The HPU also advised antibiotic treatment and prophylaxis for the case and household contacts as well as screening nose and throat swabs and ascertainment of their immunisation histories.

The case was prescribed a 14-day course of clarithromycin (500 mg twice a day). Three household contacts were identified by the family doctor: the parents and a younger sibling. Close household contacts were prescribed a seven-day course of erythromycin (dose based on their age and weight). The family doctor confirmed that the case had received a primary immunisation course at two, three and four months of age, but there was no record of a pre-school booster. The school records showed that the case had also received a dose of diphtheria-containing vaccine as part of the schoolleavers' immunisation schedule in January 2009.

The case was clinically assessed by the family doctor, in consultation with HPU and the infectious disease physician at the local hospital following confirmation of the results and found to be well, not requiring readmission to hospital. The family doctor was advised to offer a convalescent diphtheria booster vaccination to the case after January 2010 (i.e. 12 months after the last dose).

\section{Contact investigations}

It was assessed that the case may have been infectious since 19 December 2009 (i.e. seven days before onset of illness) and could have remained infectious until 8 January 2010 (i.e two weeks after onset of symptoms) which coincided with the start of antibiotic treatment with clarithromycin.

National guidelines were followed to identify close contacts at highest risk [2]. The contact investigations in this case included:

- Contacts in the hospital between 31 December 2009 and 4 January 2010. During the four days the case was hospitalised, they were cared for in a sixbedded open section of the ward. Investigations included risk assessment for hospital staff, patients and their visitors who stayed in the ward overnight.

- Contacts in the community between 19 December 2009 and 8 January 2010

In-depth interviews with the case and family confirmed that there was no recent travel history or contact with overseas visitors. There was no contact with other known cases.

\section{Hospital contacts}

A member of the HPU team was able to undertake a risk assessment with the hospital infection control team. Twelve healthcare workers were identified to have had contact with the case - none of them were involved in any aerosol generating procedures. All were fully immunised and were advised on self-surveillance. Two doctors involved with the case's care reported sore throats. They were swabbed, treated with clarithromycin and excluded from work until results of swabs were reported; both were negative.

Two patients were staying in the same section of the ward at the same time as the case: one child, whose mother stayed overnight, was in the same bay; mother and child were swabbed and given prophylactic antibiotics. The second child was in an adjacent bay and therefore not a high risk contact. The family doctors of both children and the parents were contacted and informed about their patients' exposure and advised to offer a booster dose of diphtheria-containing vaccine if the last dose received dated back more than 12 months. Both families were also informed about their exposure, and received health protection advice on signs and symptoms of diphtheria and their need for further immunisation.

\section{Household and close contacts}

Following the initial risk assessment of the case, three close household contacts (parents and a sibling) were identified. In-depth interviews with the case and family members identified a further seven contacts (family and friends), two of whom were considered to be close contacts. They were swabbed and provided with prophylaxis and were advised on signs and symptoms of the disease. All contacts were sent an advice sheet, and the family doctors were advised to review immunisa-

TABLE

Isolates of toxigenic corynebacteria, England and Wales, 1986-2009a [3]

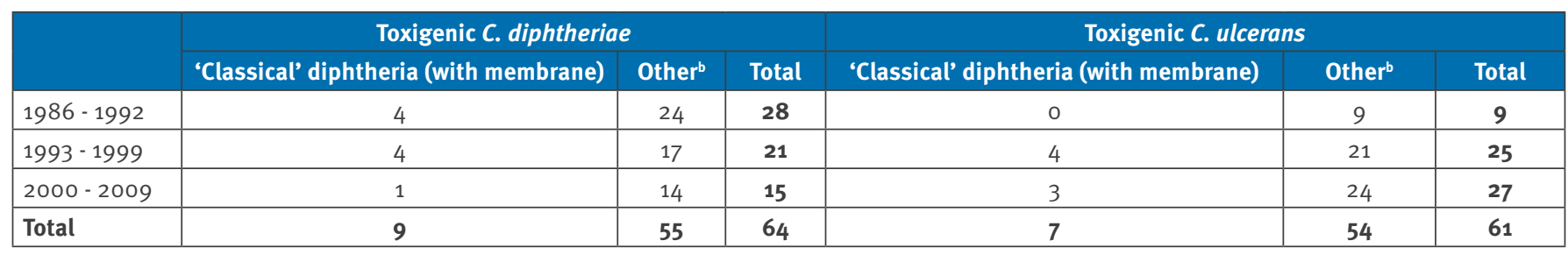

a Data for 2009 are provisional

${ }^{b}$ Other includes respiratory diphtheria without a membrane, cutaneous diphtheria, and asymptomatic carriers 
tion history and offer a booster dose if none had been received in the previous 12 months.

No school or work contacts were identified as the case was not at school during the infectious or incubation period. No other contacts were identified. All swabs from contacts were negative.

\section{Discussion}

Classical respiratory diphtheria is rare in the UK, and between 1986 and 2007 only eight cases of classical respiratory diphtheria caused by toxigenic C. diphtheriae were reported, all of whom had a history of travel to endemic countries. Countries with endemic diphtheria are Algeria, Angola, Egypt, Niger, Nigeria, Sudan, and sub-Saharan countries, Bolivia, Brazil, Colombia, Dominican Republic, Ecuador, Haiti, and Paraguay, Afghanistan, Bangladesh, Bhutan, Burma (Myanmar), Cambodia, China, India, Indonesia, Laos, Malaysia, Mongolia, Nepal, Pakistan, Papua New Guinea, Philippines, Thailand, and Vietnam, Iran, Iraq, Saudi Arabia, Syria, Turkey, and Yemen, Albania, Russia, and countries of the former Soviet Union [7]. In 2008 a fatal case of laryngeal diphtheria, caused by a toxigenic $C$. diphtheriae var mitis strain, was reported in London in an unimmunised child [4]. There was no history of recent travel and although one close contact had travelled to Africa a month before the child became unwell, the throat swab taken from this contact was negative.

The source of infection for the case discussed in this paper remains unknown; however, the association of the ribotype with Belarus implies that it may have been imported. There was no contact with known cases of diphtheria and no close contacts were identified as carriers of toxigenic $C$. diphtheriae. The case had no travel history, no contact with anyone who had recently travelled, and no recent contact with animals.

The case presented here was partially immunised against diphtheria, which may explain the less severe disease and non-classical presentation (i.e. lack of a pseudomembrane). While the incomplete vaccination did not protect the case against the infection, it probably protected them against the effects of toxin produced by the bacteria. Mild diphtheria is usually only diagnosed following laboratory confirmation of a toxigenic strain of corynebacteria. Throat swabs are routinely tested for corynebacteria at the hospital this case attended, but most UK laboratories will only screen for the organism if there is a clinical indication of diphtheria, and or contact with a known case. Other mild diphtheria cases therefore may be missed.

In this case there was some delay in the diagnosis of diphtheria. This was partly due to the low level of clinical suspicion as the disease is rare in the UK, a pseudo-membrane was not observed and the case was relatively well. Identification of EBV and a positive result for group C beta-haemolytic Streptococcus also complicated the diagnosis.
Some children may remain susceptible to diphtheria, particularly in London, as immunisation uptake rates are below the recommended level of $95 \%$ by two years of age. The most recent immunisation coverage data for London (October to December 2009) for children completing their primary immunisation by their second birthday in that quarter was $90.8 \%$, compared with $95.3 \%$ in England [8]. However, the uptake rate for children who received their pre-school booster by their fifth birthday, evaluated in the same quarter was only $69.9 \%$ in London and $84.3 \%$ in England.

\section{Conclusions}

Diphtheria can occur in children and adults with incomplete immunisation history, in whom the disease can be difficult to diagnose. Investigation of these cases can have considerable implications for health services. The case presented here required extensive investigations and risk assessment in hospital and community, which identified nine high risk contacts who received prophylactic antibiotic. Furthermore it highlights the need for ongoing work to improve vaccine uptake rates to ensure children receive all scheduled vaccinations. This case report also emphasises the importance of maintaining microbiological surveillance, expertise and awareness of these organisms among public health specialists, microbiologists and clinicians.

\section{Acknowledgements}

We would like to acknowledge the GP of the case, staff at the Whittington Hospital London and finally the case and family who kindly consented to this article being written.

\section{References}

1. Heymann DL, editor. Control of Communicable Diseases Manual. 19th Edition. Washington: American Public Health Association; 2008.

2. Bonnet JM, Begg NT. Control of diphtheria guidance for consultants in communicable disease control. Commun Dis Public Health. 1999;2(4):242-9. Available from: http://www. hpa.org.uk/web/HPAwebFile/HPAweb_C/1194947407702

3. Health Protection Agency 2010. Isolates of toxigenic corynebacteria England and Wales 1986-2009. Available from: http://www.hpa.org.uk/webw/HPAweb\&HPAwebStandard/HPA web_C/1195733776809? $p=1191942153427$

4. Health Protection Agency. Death in a child infected with toxigenic Corynebacterium diphtheriae in London. Health Protection Report 2008;19(2). Available from: http://www.hpa. org.uk/hpr/archives/2008/news1908.htm\#diph

5. Department of Health. Routine childhood immunisation programme 2009. United Kingdom Department of Health: 2010 18 January. Available from: http://www.immunisation.nhs.uk/ Immunisation Schedule

6. Efstratiou A, George RC. Laboratory guidelines for the diagnosis of infections caused by Corynebacterium diphtheriae and Corynebacterium ulcerans. World health Organization. Comm Dis Public Health, 1999;2(4):250-7.

7. Centers for Disease Control and Prevention (CDC). CDC Health Information for International Travel 2010. Brunette G, Kozarsky P, Magill A, Shlim D (editors). 1st ed.: Mosby; 2009. Available from: http://wwwnc.cdc.gov/travel/yellowbook/2010/ chapter-2/diphtheria.aspx

8. Health Protection Agency. COVER programme: October to December 2009. Health Protection Report HPR Wkly [Internet] 2010 [cited 26 March 2010]; 4: Immunisation. Available from: http://www.hpa.org.uk/Topics/InfectiousDiseases/ InfectionsAZ/VaccineCoverageAndCOVER 